OPEN ACCESS

Edited by:

Paolo Bonanni,

University of Florence, Italy

Reviewed by:

Amjad Khan

Quaid-i-Azam University, Pakistan

Danilo Buonsenso,

Catholic University of the Sacred

Heart, Italy

*Correspondence:

Caterina Rizzo

caterina1.rizzo@opbg.net

Specialty section:

This article was submitted to

Pediatric Infectious Diseases,

a section of the journal

Frontiers in Pediatrics

Received: 23 October 2020 Accepted: 12 April 2021

Published: 11 May 2021

Citation:

Rizzo C, Loconsole D, Pandolfi E, Ciofi Degli Atti ML, van Summeren J, Paget J, Russo L, Campagna I,

Croci I, Gesualdo F, Concato C, Linardos G, Bartolucci V, Ciampini S, Muda AO, Raponi M and Chironna M (2021) Sars-Cov2 Not Detected in a

Pediatric Population With Acute

Respiratory Infection in Primary Care in Central and Southern Italy From November 2019 to Early March 2020.

Front. Pediatr. 9:620598.

doi: 10.3389/fped.2021.620598

\section{Sars-Cov2 Not Detected in a Pediatric Population With Acute Respiratory Infection in Primary Care in Central and Southern Italy From November 2019 to Early March 2020}

Caterina Rizzo ${ }^{1 *}$, Daniela Loconsole ${ }^{2}$, Elisabetta Pandolfi ${ }^{3}$, Marta Luisa Ciofi Degli Atti ${ }^{1}$, Jojanneke van Summeren ${ }^{4}$, John Paget ${ }^{4}$, Luisa Russo ${ }^{3}$, Ilaria Campagna ${ }^{3}$, Ileana Croci ${ }^{3}$, Francesco Gesualdo ${ }^{3}$, Carlo Concato ${ }^{5}$, Giulia Linardos ${ }^{5}$, Veronica Bartolucci ${ }^{2}$, Sara Ciampini ${ }^{6}$, Andrea Onetti Muda ${ }^{7}$, Massimiliano Raponi ${ }^{8}$ and Maria Chironna ${ }^{2}$ on behalf of RSV ComNet Collaborators

${ }^{1}$ Clinical Pathways and Epidemiology, Bambino Gesù Children's Hospital, IRCCS, Rome, Italy, ${ }^{2}$ Department of Biomedical Science and Human Oncology, University of Bari, Bari, Italy, ${ }^{3}$ Multifactorial Disease and Complex Disease Area, Bambino Gesù Children Hospital, Rome, Italy, ${ }^{4}$ Nivel, Netherlands Institute for Health Services Research, Utrecht, Netherlands, ${ }^{5}$ Virology Unit, Laboratory Department, Bambino Gesù Children's Hospital, Rome, Italy, ${ }^{6}$ Public Health Service, Local Health Authority Rome 1, Rome, Italy, ${ }^{7}$ Department of Laboratories, Bambino Gesù Children's Hospital, Rome, Italy, ${ }^{8}$ Medical Direction, Bambino Gesù Children's Hospital IRCCS, Rome, Italy

Background: In December 2019, a novel coronavirus named SARS-CoV-2 started circulating in China and this led to a major epidemic in Northern Italy between February and May 2020. Young children (aged $<5$ years) seem to be less affected by this coronavirus disease (COVID-19) compared to adults, although there is very little information on the circulation of this new virus among children in Italy. We retrospectively tested nasopharyngeal swabs for SARS-CoV-2 in samples collected in young children between November, 2019 and March, 2020 in the context of the RSV ComNet study.

Methods: Two networks of primary care pediatricians in Lazio (Central Italy) and Puglia (Southern Italy) collected nasopharyngeal swabs from children, aged $<5$ years, presenting with symptoms for an acute respiratory infection (ARI). The RSV ComNet study is a multicenter study implemented to estimate the burden of RSV in young children (aged $<5$ years) in the community. Swabs were sent to a central reference laboratory and tested for 14 respiratory viruses through RT-PCR. All collected samples were retrospectively tested for SARS-CoV-2 using RT-PCR (Istituto Superiore di Sanità protocol).

Results: A total of 293 children with ARI were identified in the two participating networks. The highest number of cases were recruited in weeks 51/2019 and $3 / 2020$. The majority of patients (57\%) came from the Lazio region. All of the 293 samples tested negative for SARS-Cov2. Rhinovirus was the most frequently detected virus (44\%), followed by RSV (41\%) and influenza viruses (14\%). 


\begin{abstract}
Conclusions: Our study shows that in Lazio (a region of intermediate SARS-COV-2 incidence) and Puglia (a region of low incidence), the SARS-Cov2 virus did not circulate in a sample of ARI pediatric cases consulting primary care pediatricians between November 2019 and March 2020.
\end{abstract}

Keywords: SARS-CoV2, ARI, RSV, pediatric cases, epidemiology, primary care

\section{INTRODUCTION}

In December 2019, a novel coronavirus named severe acute respiratory syndrome coronavirus 2 (SARS-CoV-2), causing COVID-19 (coronavirus disease 2019), started circulating in China and subsequently spread in many countries (1). On the 11th of March, 2020, COVID-19 was declared a pandemic by WHO (2) and on the 30th of January, 2020, Italy reported the first 2 cases of SARS-CoV-2 infection (3-5), both with a history of travel to Wuhan, China (5). The first Italian patient with COVID19 was diagnosed on February 21, 2020, a 38-year-old man hospitalized at Codogno Hospital, Lodi, in northern Italy, with no travel history to areas where the virus was already isolated or link to a probable or confirmed COVID-19 case (6). On the same day, another outbreak of COVID-19 was reported in Vò Euganeo (Padua) in the Veneto region, with the first death reported in a 78-year-old man hospitalized in Padua (6). Before this date, in Italy only three cases of SARS-CoV2 infection were identified, all with travel history to Wuhan. Subsequently, extensive and ongoing transmission was identified in several municipalities of the Lombardy region (7). Thereafter, case counts, and death tolls rapidly increased, with the majority of cases in northern Italy with few cases reported in the rest of the country. The Italian government recommended strict physical distancing measures closing 10 municipalities in the Lodi Province (Lombardy) and one in the Padua province (Veneto) on the 23rd of February 2020. This culminated in a national lock-down on the 11th of March $2020(8,9)$ that resulted in a reduction of the incidence of the COVID19 pandemic (10). However, as already reported by some countries, measures implemented during the lockdown results in a substantial drop in the number of children completing their vaccination schedule, which could lead to possible further outbreaks of vaccine-preventable diseases (11-14).

Some studies conducted suggest that SARS-CoV-2 might entered Italy between December and early February 2020 a scenario that is compatible with observations in other European (15-18). Very little information is available in the literature on the circulation of SARS-Cov2 in the pediatric population. Recently, one study conducted in Italy identified in sera collected from September 2019 to February 2020 seropositive adults (14.2\% in September and $16.3 \%$ in October) with IgG or IgM antibodies, or both (19). A recent study demonstrate the presence of a confirmed SARS-CoV2 child $\sim 3$ months before the first identified coronavirus disease case in Italy (20). However, children seem to be less affected by COVID19 than adults are and a large cohort study conducted in Italy in a network of ED showed that hospitalized children may have rare but serious and life-threatening presentations (21). In Europe, as of 13 May 2020, only $0.7 \%$ of the 576024 laboratory-confirmed cases reported to the ECDC was $<4$ year of age (22). The objective of our study was to characterize the circulation of SARS-CoV-2 among Italian children at the beginning of the COVID-19 epidemic in Italy. To meet this objective, we retrospectively looked for SARS-CoV-2 in nasopharyngeal aspirate samples collected among children aged $<5$ years, presenting to their primary care pediatricians with symptoms of an acute respiratory infection (ARI). The samples had been collected between November, 2019 and March, 2020 in the context of a study on the epidemiology of the Respiratory Syncytial Virus (RSV).

\section{METHODS}

\section{Study Population}

The organization of pediatric primary care in Italy is one in which young children $(<6$ years old $)$ are generally cared for by a pediatrician and school-age children (6-14 years old) have the option to see either a pediatrician or a general practitioner. We carried out our study using two networks of primary care pediatricians (PCP) - one in the Lazio Region (Central Italy) and one in the Puglia Region (Southern Italy). The participating pediatricians collected the nasopharyngeal swabs from children, younger than 5 years, presenting at their office with ARI symptoms, between week 47/2019 (mid-November 2019) and week 11/2020 (early March).

\section{ARI Case Definition}

The ARI case definition was based on the WHO definition for community-based RSV surveillance (23) and included the following criteria:

(1) Acute - defined as a sudden onset of symptoms;

(2) Respiratory infection - defined as having at least one of the following: shortness of breath, cough, sore throat, coryza;

(3) Clinician's judgement that the illness is due to an infection.

Note: Point (3) was added to the definition by the RSV ComNet research team.

\section{Study Design and Procedure}

The study was conducted in the framework of a multicenter study to estimate the burden of RSV in children $<5$ years of age in the community (the RSV-ComNet, see: https:// nivel.nl/en/RSVComNet). In this study, swabs were sent to a regional laboratory and tested for 16 respiratory viruses 


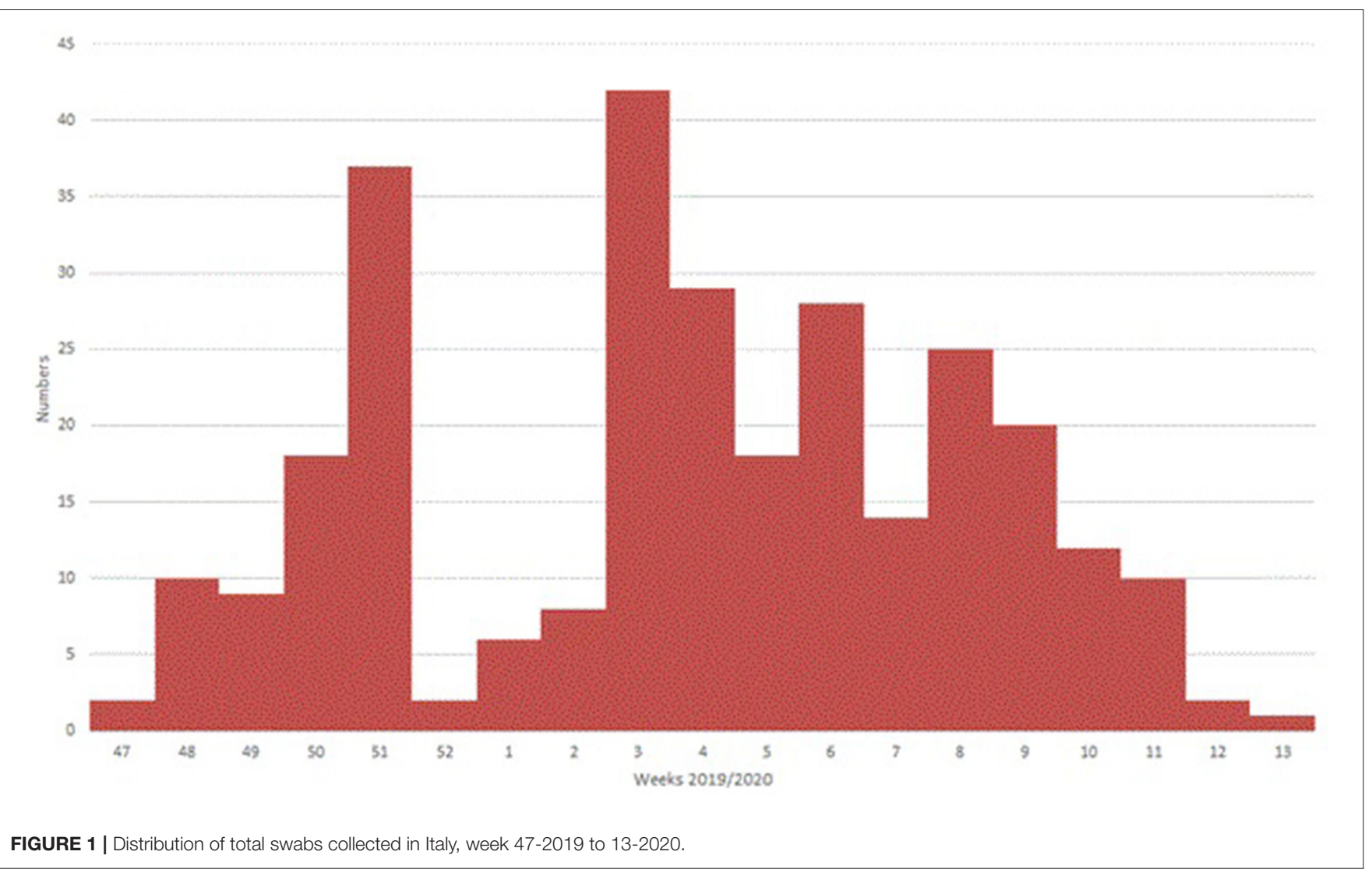

(including RSV A and $\mathrm{B}$, influenza virus $\mathrm{A}$ and $\mathrm{B}$, human coronavirus OC43, 229E, NL-63 and HUK1, adenovirus, hRV, parainfluenza virus 1-2-3-4, human metapneumovirus-hMPV and human bocavirus-hBoV) through RT-PCR. Informed consent was obtained from parents of enrolled children. At enrollment, PCPs collected preliminary information for each of the recruited patients, including demographic data (birth date, sex, date of swabbing), date of onset of clinical symptoms, and clinical picture at enrollment. Descriptive statistics were used to describe the patient demographics, and clinical symptoms. All data were analyzed using STATA $13 \AA$.

\section{Laboratory Analysis}

After being collected and analyzed, all samples were frozen at $-80^{\circ} \mathrm{C}$ in a Thermo ScientificTM $-86^{\circ} \mathrm{C}$ freezer and stored in case of need for further analysis. In May-June 2020, all previously collected samples were tested for SARS-CoV2 , independently from the previous test results. Nucleic acid was extracted using the STARMag Universal Cartridge kit (Seegene) on the automated Nimbus IV platform. SARS-CoV2 RT-PCR was performed on CFX96 (Bio Rad Laboratories) with AllplexTM 2019 n-CoV Assay according to the Istituto Superiore di Sanità protocol (14), with the targets being the E gene, encoding the envelope protein of Sarbecovirus, RdRp (RNA-dependent RNA polymerase) and $\mathrm{N}$ (nucleocapside) genes specific for CoV-2019.

\section{Ethical Approval}

The Medical Ethical Committee of OPBG Medical Center (Italy) approved the original study (prot. 1936_OPBG_2019), and subsequently waived the study for the additional SARS-CoV-2 testing on the 12th May 2020 (prot. N 525).

\section{RESULTS}

We recruited ARI cases from two municipalities located in two Italian regions: Rome in the Lazio region and Bari in the Puglia region. The population of the Roma municipality accounts for $7.2 \%$ of the Italian population, and the population of Bari municipality accounts for $2.1 \%$ of the Italian population. The total population of children younger than 5 years of age in the two participating municipalities accounted for $5 \%$ of the National population aged $0-5$ years. The study involved 12 pediatricians from the Lazio region and 12 pediatricians in the Puglia region.

A total of 293 patients with ARI symptoms presenting to their primary care pediatricians were recruited in the two participating networks (24 participating pediatricians). The highest number of cases were enrolled in week 51/2019 and 3/2020 (Figure 1). The majority of ARI patients $(168 ; 57 \%)$ came from the Lazio region, and 125 (43\%) were from the Puglia region. Table 1 presents a description of demographic and clinical characteristics of the enrolled patients. Most patients 130 (44\%) were younger than 1 year of age, 70 (24\%) belonged to the 12-24 months age group and 93 (32.7\%) were $24-36$ months old. The median 
TABLE 1 | Description of ARI cases characteristics.

\begin{tabular}{|c|c|c|c|c|c|c|}
\hline \multirow[t]{2}{*}{ Characteristic } & \multicolumn{2}{|c|}{$\begin{array}{l}\text { Total } \\
(n=293)\end{array}$} & \multicolumn{2}{|c|}{$\begin{array}{l}\text { Lazio } \\
(n=168)\end{array}$} & \multicolumn{2}{|c|}{$\begin{array}{l}\text { Puglia } \\
(n=125)\end{array}$} \\
\hline & $n$ & $\%$ & $n$ & $\%$ & $n$ & $\%$ \\
\hline \multicolumn{7}{|l|}{ Region } \\
\hline Lazio & 168 & 57.3 & & & & \\
\hline Puglia & 125 & 42.7 & & & & \\
\hline Sex $=$ male & 159 & 54.3 & 87 & 51.8 & 72 & 57.6 \\
\hline \multicolumn{7}{|l|}{ Age group (months) } \\
\hline $0-12$ & 130 & 44.4 & 69 & 41.1 & 61 & 48.8 \\
\hline $13-24$ & 70 & 23.9 & 48 & 28.6 & 22 & 17.6 \\
\hline $25-60$ & 93 & 31.7 & 51 & 30.4 & 42 & 33.6 \\
\hline Pre-maturity & 10 & 3.5 & 5 & 3.1 & 5 & 4.0 \\
\hline \multicolumn{7}{|c|}{ Presence of chronic condition } \\
\hline Respiratory disease & 4 & 1.4 & 1 & 0.6 & 3 & 2.4 \\
\hline Malnutrition & 1 & 0.4 & 1 & 0.6 & - & - \\
\hline Immunocompromised & 0 & - & - & - & - & - \\
\hline Others & 4 & 1.4 & 1 & 0.6 & 3 & 2.4 \\
\hline \multicolumn{7}{|l|}{ Symptoms } \\
\hline Shortness of breath & 177 & 61.7 & 91 & 56.2 & 86 & 68.8 \\
\hline Cough & 286 & 97.6 & 162 & 96.4 & 124 & 99.2 \\
\hline Sore throat & 122 & 41.8 & 88 & 52.7 & 34 & 27.2 \\
\hline Coryza & 251 & 86.3 & 153 & 92.2 & 98 & 78.4 \\
\hline
\end{tabular}

number of days between disease onset and swabbing was 2 (IQR 1-3.5). Cough was the most frequently reported symptom (98\%), followed by coryza (86\%) and shortness of breath (62\%). Very few cases were affected by underlying conditions (Table 1). Eighteen cases presented to the ED after the pediatricians visit and seven were hospitalized. All samples tested negative for SARS-CoV-2. Rhinovirus was the most frequently detected virus (44\%) followed by RSV (41\%) and influenza viruses (14\%) (Table 2). A high number of coinfections was observed (215, corresponding to $73.4 \%$ of all enrolled patients). Most frequently isolated coinfections were Rhinovirus and Bocavirus $(N=8)$, Rhinovirus + Adenovirus $(N=7)$ and Rhinovirus + Coronavirus OC43 $(N=6)$.

\section{DISCUSSION}

We show that no SARS-Cov2 infections were detected in nasopharyngeal swabs in a sample of 293 children with ARI symptoms aged 0-5 years, enrolled between November 2019 and early March 2020 in the Italian regions of Lazio and Puglia. COVID-19 caused by SARS-CoV-2 was first reported in December 2019, in the city of Wuhan in the province of Hubei, China, in people who had visited a seafood market (24). By the time the real potential of its pathogenicity was realized, it had spread to many regions of China, other Asian countries, European countries, the United States etc. and by April 20, 2020, it had spread to 185 countries all over the world (25). By February 21,2020 , there were 47 confirmed cases of COVID-19 in the European Region i.e., France (12 positive cases), Germany (16 cases), Belgium (1 case), Finland (1 case), Italy (3 cases), Spain
TABLE 2 | Distribution of ARI cases by type of virus and by age group.

\begin{tabular}{|c|c|c|c|c|c|c|c|c|}
\hline \multirow{2}{*}{\multicolumn{2}{|c|}{ Total }} & \multicolumn{6}{|c|}{ Age group (months) } & \multirow[b]{3}{*}{ D } \\
\hline & & \multicolumn{2}{|c|}{$0-12$} & \multicolumn{2}{|c|}{$13-24$} & \multicolumn{2}{|c|}{$25-60$} & \\
\hline$n$ & $\%$ & $n$ & $\%$ & $n$ & $\%$ & $n$ & $\%$ & \\
\hline 128 & 43.7 & 60 & 46.2 & 32 & 45.7 & 36 & 38.7 & 0.848 \\
\hline 119 & 40.6 & 53 & 40.8 & 26 & 37.1 & 40 & 43 & 0.751 \\
\hline 30 & 10.2 & 6 & 4.6 & 11 & 15.7 & 13 & 14 & 0.052 \\
\hline 24 & 8.2 & 6 & 4.6 & 10 & 14.3 & 8 & 8.6 & 0.172 \\
\hline 21 & 7.2 & 7 & 5.4 & 6 & 8.6 & 8 & 8.6 & 0.786 \\
\hline 20 & 6.8 & 7 & 5.4 & 7 & 10 & 6 & 6.5 & 0.736 \\
\hline 20 & 6.8 & 8 & 6.2 & 5 & 7.1 & 7 & 7.5 & 0.955 \\
\hline 19 & 6.5 & 11 & 8.5 & 3 & 4.3 & 5 & 5.4 & 0.751 \\
\hline 11 & 3.8 & 4 & 3.1 & 4 & 5.7 & 3 & 3.2 & 0.849 \\
\hline 9 & 3.1 & 2 & 1.5 & 2 & 2.9 & 5 & 5.4 & 0.499 \\
\hline 6 & 2 & 2 & 1.5 & 4 & 5.7 & 0 & - & 0.131 \\
\hline 6 & 2 & 3 & 2.3 & 0 & - & 3 & 3.2 & 0.613 \\
\hline 5 & 1.7 & 1 & 0.8 & 3 & 4.3 & 1 & 1.1 & 0.397 \\
\hline 2 & 0.7 & 0 & - & 1 & 1.4 & 1 & 1.1 & 0.711 \\
\hline 2 & 0.7 & 2 & 1.5 & 0 & - & 0 & - & 0.579 \\
\hline 0 & - & 0 & - & 0 & - & 0 & - & - \\
\hline
\end{tabular}

( 2 cases), Russia ( 2 cases), Sweden (1 case) and UK (9 cases). By March 6, 2020, there were 5,544 COVID-19 cases and 159 deaths in the EU and the UK (17 cases) (3). Of all the European countries, Italy was the worst affected in the initial stage. As of late March 2020, all Italian regions and Autonomous Provinces had reported at least one locally acquired case of COVID-19. In particular, there were high incidence regions with sustained local transmission (mainly in the north), low incidence regions (like Puglia) with limited but growing numbers of locally acquired cases of infection and regions with intermediate incidence (like Lazio) (26). Some studies have speculated that SARS-CoV-2 may have been circulating before the first recorded local cases in several countries (15-17). The majority of these studies were phylogenetic, serological or retrospective analyses of mainly adults cases $(15,27-31)$. While some were the result of modeling studies speculating that in some parts of the country, such as in central (Lazio) and southern regions (Puglia), transmission in adults was largely undetected until the first days of March (21). Nevertheless, few information on the circulation of SARS-CoV2 in the pediatric population in Italy in the community is available, to date (21).

Our study could have some potential limitations. Firstly, it has been argued that nasopharyngeal swab is not the gold standard for diagnosis of SARS-CoV2 in children $(32,33)$. In our study we swabbed only symptomatic ARI children within 2 days (IQR 1-3.5), on average, from symptoms onset, and, therefore, this could have increased nasopharyngeal swab sensitivity. Another possible limitation is due to the fact that is difficult to generalize our results to the wider population, as we only focused on the pediatric population. However, our study was concentrated only on ARI symptomatic cases presenting to their pediatricians in primary care. 
We found a very high proportion of coinfections in our sample with most frequently isolated coinfections being Rhinovirus + Bocavirus, followed by Rhinovirus + Adenovirus and Rhinovirus + Coronavirus OC43. As reported in a recent meta-analysis (34) we observed that RSV and Adenovirus were mostly detected as single infections. This proportion is higher to what observed in previous studies and is probably due to the fact that the number of co-viruses tested in Italy was larger in comparison to the Netherlands, but also larger compared to other published studies (35).

Our study suggests that, based on a sample of ARI pediatric cases consulting primary care pediatricians in low and intermediate incidence areas of Italy from November 2019 to March 2020, the SARS-Cov2 virus did not circulate in children aged $<$ then 5 years. These findings are consistent with the epidemiological situation of COVID-19 in Italy, as the increase in the incidence was observed later in the regions where this study was conducted compared to the northern regions where the first Italian cases occurred and which had the greatest impact (6).

\section{DATA AVAILABILITY STATEMENT}

The raw data supporting the conclusions of this article will be made available by the authors, without undue reservation.

\section{ETHICS STATEMENT}

The studies involving human participants were reviewed and approved by The Medical Ethical Committee of OPBG Medical Center (Italy) approved the original study

\section{REFERENCES}

1. Zhu N, Zhang D, Wang W, Li X, Yang B, Song J, et al. A novel coronavirus from patients with pneumonia in China, 2019. N Engl J Med. (2020) 382:72733. doi: 10.1056/NEJMoa2001017

2. WHO. WHO Director-General's Opening Remarks at the Media Briefing on COVID-19. (2020). Available online at: https://www.who.int/directorgeneral/speeches/detail/who-director-general-s-opening-remarks-at-themedia-briefing-on-covid-19--11-march-2020 (accessed November 16, 2020)

3. Lescure F-X, Bouadma L, Nguyen D, Parisey M, Wicky P-H, Behillil S, et al. Clinical and virological data of the first cases of COVID-19 in Europe: a case series. Lancet Infect Dis. (2020) 20:697-706. doi: 10.1016/S1473-3099(20)30200-0

4. Spiteri G, Fielding J, Diercke M, Campese C, Enouf V, Gaymard A, et al. First cases of coronavirus disease 2019 (COVID-19) in the WHO European region, 24 January to 21 February 2020. Euro Surveill. (2020) 25:2000178. doi: 10.2807/1560-7917.ES.2020.25.9.2000178

5. Giovanetti M, Benvenuto D, Angeletti S, Ciccozzi M. The first two cases of 2019-nCoV in Italy: where they come from? J Med Virol. (2020) 92:51821. doi: 10.1002/jmv.25699

6. Indolfi C, Spaccarotella C. The outbreak of COVID-19 in Italy. JACC Case Rep. (2020) 2:1414-8. doi: 10.1016/j.jaccas.2020.03.012

7. Guzzetta G, Poletti P, Ajelli M, Trentini F, Marziano V, Cereda D, et al. Potential short-term outcome of an uncontrolled COVID-19 epidemic in Lombardy, Italy, February to March 2020. Eurosurveillance. (2020) 25:2000293. doi: 10.2807/1560-7917.ES.2020.25.12.2000293

8. Decreto del presidente del consiglio dei ministri (2020). Available online at: https://www.trovanorme.salute.gov.it/norme/dettaglioAtto?id=73643 (accessed November 16, 2020). (prot. 1936_OPBG_2019), and subsequently waived the study for the additional SARS-CoV-2 testing on the 12th May 2020 (prot. $\mathrm{N}$ 525). Written informed consent to participate in this study was provided by the participants' legal guardian/next of kin.

\section{AUTHOR CONTRIBUTIONS}

CR conceived the study, coordinated the study, and wrote the article. EP wrote the article and contributed to the interpretation of results. $\mathrm{ICr}$ performed the statistical analysis. DL, LR, ICa, FG, CC, GL, VB, and SC coordinated the study at the local level and revised the draft article. JP and JS supported the coordination of the study at the international level and revised the draft article. MLC, AM, MR, and MC revised the draft article and contributed to the interpretation of results. All authors contributed to the article and approved the submitted version.

\section{FUNDING}

The authors declare that this study received funding from Sanofi Pasteur \& AstraZeneca. The funder was not involved in the study design, collection, analysis, interpretation of data, the writing of this article or the decision to submit it for publication.

\section{ACKNOWLEDGMENTS}

The study team is very grateful to all parents, patients, pediatricians, and laboratory teams who have contributed to the study. We also thank the RSV Com Net collaborators.

9. Decreto del presidente del consiglio dei ministri (2020). Available online at: https://www.trovanorme.salute.gov.it/norme/dettaglioAtto?id=73196\& articolo=6 (accessed November 16, 2020).

10. Guzzetta G, Riccardo F, Marziano V, Poletti P, Trentini F, Bella A, et al. Impact of a nationwide lockdown on SARS-CoV-2 transmissibility, Italy. Emerg Infect Dis. (2021) 27:267-70. doi: 10.3201/eid2701.202114

11. WHO and UNICEF warn of a decline in vaccinations during COVID19 (2021). Available online at: https://www.who.int/news/item/15-072020-who-and-unicef-warn-of-a-decline-in-vaccinations-during-covid-19 (accessed January 27, 2021).

12. Khan A, Bibi A, Sheraz Khan K, Raza Butt A, Alvi HA, Zahra Naqvi A, et al. Routine pediatric vaccination in Pakistan during Covid19: how can healthcare professionals help? Front Pediatr. (2020) 8:613433. doi: 10.3389/fped.2020.613433

13. Saxena S, Skirrow H, Bedford H. Routine vaccination during covid-19 pandemic response. BMJ. (2020) 369:m2392. doi: 10.1136/bmj.m2392

14. Carias C, Pawaskar M, Nyaku M, Conway JH, Roberts CS, Finelli L, et al. Potential impact of COVID-19 pandemic on vaccination coverage in children: a case study of measles-containing vaccine administration in the United States (US). Vaccine. (2020) 39:1201-4. doi: 10.1016/j.vaccine.2020.11.074

15. Lai A, Bergna A, Caucci S, Clementi N, Vicenti I, Dragoni F, et al. Molecular tracing of SARS-CoV-2 in Italy in the first three months of the epidemic. Viruses. (2020) 12:798. doi: 10.3390/v12080798

16. Onder G, Rezza G, Brusaferro S. Case-fatality rate and characteristics of patients dying in relation to COVID-19 in Italy. JAMA. (2020) 323:17756. doi: 10.1001/jama.2020.4683

17. Gámbaro F, Behillil S, Baidaliuk A, Donati F, Albert M, Alexandru $A$, et al. Introductions and early spread of SARS-CoV-2 in France, 24 January to 23 March 2020. Eurosurveillance. (2020) 25:2001200. doi: 10.2807/1560-7917.ES.2020.25.26.2001200 
18. WHO. WHO Technical Meeting on Piloting RSV Surveillance Based on the Global Influenza Surveillance and Response System. WHO (2020). Available online at: http://www.who.int/influenza/resources/publications/Technical Meeting_RSV_Pilot/en/ (accessed September 2, 2020)

19. Apolone G, Montomoli E, Manenti A, Boeri M, Sabia F, Hyseni I, et al. Unexpected detection of SARS-CoV-2 antibodies in the prepandemic period in Italy. Tumori. (2020) 0300891620974755. doi: 10.1177/0300891620974755

20. Amendola A, Bianchi S, Gori M, Colzani D, Canuti M, Borghi E, et al. Evidence of SARS-CoV-2 RNA in an oropharyngeal swab specimen, Milan, Italy, Early December 2019. Emerg Infect Dis J. 27:648-50. doi: 10.3201/eid2702.204632

21. Parri N, Lenge M, Cantoni B, Arrighini A, Romanengo M, Urbino A, et al. COVID-19 in 17 Italian pediatric emergency departments. Pediatrics. (2020) 146:e20201235. doi: 10.1542/peds.2020-1235

22. Rapid risk assessment: Paediatric inflammatory multisystem syndrome and SARS -CoV-2 infection in children. European Centre for Disease Prevention and Control (2020). Available online at: https://www.ecdc.europa.eu/en/ publications-data/paediatric-inflammatory-multisystem-syndrome-andsars-cov-2-rapid-risk-assessment (accessed 21 January 2021)

23. WHO. RSV Surveillance Case Definitions. WHO (2020). Available online at: http://www.who.int/influenza/rsv/rsv_case_definition/en/ (accessed October 20, 2020).

24. Li Q, Guan X, Wu P, Wang X, Zhou L, Tong Y, et al. Early transmission dynamics in Wuhan, China, of novel coronavirus-infected pneumonia. N Engl J Med. (2020) 382:1199-207. doi: 10.1056/NEJMoa20 01316

25. Mallhi TH, Khan YH, Alotaibi NH, Alzarea AI, Tanveer N, Khan A. Celebrating eid-ul-adha in the era of the COVID-19 pandemic in Pakistan: potential threats and precautionary measures. Clin Microbiol Infect. (2020) 26:1714-5. doi: 10.1016/j.cmi.2020.07.019

26. Riccardo F, Ajelli M, Andrianou XD, Bella A, Del Manso M, Fabiani $\mathrm{M}$, et al. Epidemiological characteristics of COVID-19 cases and estimates of the reproductive numbers 1 month into the epidemic, Italy, 28 January to 31 March 2020. Euro Surveill. (2020) 25:2000790. doi: 10.2807/1560-7917.ES.2020.25.49.2000790

27. Sarigül F, Doluca O, Akhan S, Sayan M. Investigation of compatibility of severe acute respiratory syndrome coronavirus 2 reverse transcriptasePCR kits containing different gene targets during coronavirus disease 2019 pandemic. Future Virol. (2020) 15:515-24. doi: 10.2217/fvl-2020-0169

28. Barreto HG, de Pádua Milagres FA, de Araújo GC, Daúde MM, Benedito VA. Diagnosing the novel SARS-CoV-2 by quantitative RT-PCR: variations and opportunities. J Mol Med. (2020) 98:1727-36. doi: 10.1007/s00109-020-01992-x
29. Shah SJ, Barish PN, Prasad PA, Kistler A, Neff N, Kamm J, et al. Clinical features, diagnostics, and outcomes of patients presenting with acute respiratory illness: a retrospective cohort study of patients with and without COVID-19. EClin Med. (2020) 27:100518. doi: 10.1016/j.eclinm.2020. 100518

30. Guan W, Ni Z, Hu Y, Liang W, Ou C, He J, et al. Clinical characteristics of coronavirus disease 2019 in China. N Engl J Med. (2020) 80:65665. doi: 10.1101/2020.02.06.20020974

31. World Health Organization. WHO Strategy to Pilot Global Respiratory Syncytial Virus Surveillance Based on the Global Influenza Surveillance and Response System (GISRS). World Health Organization (2017). Available online at: https://apps.who.int/iris/handle/10665/259853 (accessed September 2020).

32. Marino S, Ruggieri M, Falsaperla R. Is SARSCoV-2 nasopharyngeal swab still a gold standard in children? Med Hypotheses. (2020) 144:110041. doi: 10.1016/j.mehy.2020.110041

33. Buonsenso D, Valentini P, Rose CD, Pata D, Sinatti D, Speziale D, et al. Seroprevalence of anti-SARS-CoV-2 IgG antibodies in children with household exposition to adults with COVID-19: preliminary findings. medRxiv. (2020) 2020.08.10.20169912. Available online at: https://www. medrxiv.org/content/10.1101/2020.08.10.20169912v1 (accessed January 21, 2021).

34. Deslandes A, Berti V, Tandjaoui-Lambotte Y, Alloui C, Carbonnelle E, Zahar JR, et al. SARS-CoV-2 was already spreading in France in late December 2019. Int J Antimicrob Agents. (2020) 55:106006. doi: 10.1016/j.ijantimicag.2020.106006

35. Li Y, Pillai P, Miyake F, Nair H. The role of viral co-infections in the severity of acute respiratory infections among children infected with respiratory syncytial virus (RSV): a systematic review and meta-analysis. J Glob Health. (2020) 10:010426. doi: 10.7189/jogh.10.010426

Conflict of Interest: The authors declare that the research was conducted in the absence of any commercial or financial relationships that could be construed as a potential conflict of interest.

Copyright () 2021 Rizzo, Loconsole, Pandolfi, Ciofi Degli Atti, van Summeren, Paget, Russo, Campagna, Croci, Gesualdo, Concato, Linardos, Bartolucci, Ciampini, Muda, Raponi and Chironna. This is an open-access article distributed under the terms of the Creative Commons Attribution License (CC BY). The use, distribution or reproduction in other forums is permitted, provided the original author(s) and the copyright owner(s) are credited and that the original publication in this journal is cited, in accordance with accepted academic practice. No use, distribution or reproduction is permitted which does not comply with these terms. 